\title{
Divisions Panel Discussion: Astronomy for Development
}

\section{Kevin Govender ${ }^{1}$, Mary Kay Hemenway ${ }^{2}$, Anna Wolter ${ }^{3}$, Nader Haghighipour $^{4}$, Yihua Yan $^{5}$, E.F. van Dishoeck ${ }^{6}$, David Silva ${ }^{7}$ and Edward Guinan ${ }^{8}$}

${ }^{1}$ Facilitator of Panel Discussion, IAU Office of Astronomy for Development, Cape Town, South Africa

${ }^{2}$ University of Texas at Austin, USA

${ }^{3}$ INAF-Osservatorio Astronomico di Brera, Via Brera 28, 20121, Milano, Italy

${ }^{4}$ Institute for Astronomy, University of Hawaii-Manoa

${ }^{5}$ National Astronomical Observatories, Chinese Academy of Sciences, A20 Datun Road, Chaoyang District, Beijing 100012, China

${ }^{6}$ van Dishoeck, Leiden Observatory, Leiden University, the Netherlands

${ }^{7}$ National Optical Astronomy Observatory, 950 North Cherry Avenue, Tucson, AZ, 85719 USA

${ }^{8}$ Villanova University, 800 Lancaster Avenue, Villanova, PA 19085 USA

\begin{abstract}
The main purpose of this panel discussion was to encourage conversation around potential collaborations between the IAU Office of Astronomy for Development (OAD) and IAU Divisions. The discussion was facilitated by the OAD and the conversation revolved mainly around two questions: (i) What should the OAD be doing to enhance the work of the Divisions? (ii) What could the Divisions (both members and respective scientific discipline in general) contribute towards the implementation of the IAU strategic plan?
\end{abstract}

Keywords. OAD, Divisions, astronomy, development

\section{Format and Participants}

The discussion followed a relatively open format with conversation around potential collaborations between the OAD and respective IAU Divisions. Due to the large number of panellists there were only two rounds of "going-around-the-table" in turn and the rest were voluntary contributions as points arose. The participants in the discussion (representing their respective divisions) are listed in Table 1 below:

\section{Summary of discussions}

The discussion was in general quite dynamic and included input and question from the audience. Some important points are captured here and include input received by panellists by email before and after the discussion.

(i). Each Division (or area of astronomy) may produce specific resources that relate to education and development - the OAD could serve to collect, compare and disseminate these e.g. tutorials, lecture notes, assessments, outreach materials, etc.

(ii). The OAD has 9 regional offices and a large network of collaborators - this can be used to disseminate information and resources related to each Division's activities.

(iii). Divisions could consider the establishment of a small committee or single point of contact to be the interface with the OAD. 
Table 1: List of IAU Divisions and Representatives

\begin{tabular}{|c|c|}
\hline Division & Representative \\
\hline Division A Fundamental Astronomy & Anne Lemaitre \\
\hline Division B Facilities, Technologies and Data Science & David Silva \\
\hline Division C Education, Outreach and Heritage & Mary Kay Hemenway \\
\hline $\begin{array}{l}\text { Division D High Energy Phenomena and Fundamental } \\
\text { Physics }\end{array}$ & Anna Wolter \\
\hline Division E Sun and Heliosphere & Yihua Yan \\
\hline Division F Planetary Systems and Bioastronomy & Nader Haghighipour \\
\hline Division G Stars and Stellar Physics & $\begin{array}{l}\text { Ed Guinan (also represented } \\
\text { Division J) }\end{array}$ \\
\hline Division H Interstellar Matter and Local Universe & Ewine van Dishoeck \\
\hline Division J Galaxies and Cosmology & $\begin{array}{l}\text { Ed Guinan (also represented } \\
\text { Division G) }\end{array}$ \\
\hline
\end{tabular}

(iv). The OAD could develop guidelines for symposia to enhance the regional developmental benefits that could result e.g. how to maximise the impact of an outreach component, addressing issues of inclusion, increase the international participation, etc.

(v). The OAD could coordinate the development of assessment materials in order to effectively measure the impact of training activities such as schools/workshops/symposia.

(vi). There could be a collection of talks online, similar to TED talks, which are made available to growing astronomy communities around the world (it was noted by the audience though that online material could be problematic in some parts of the world)

(vii). A long discussion was held around evaluation and measurement of impact. While it was generally agreed that this was important there were concerns raised about the cost and whether some goals would be realistically achievable. This is a challenge for the OAD to apply its mind to.

(viii). OAD and the IAU Office for Astronomy Outreach (OAO) should consider Division C as a "Think Tank" for education and outreach, noting that many research based projects indicate the results of extensive evaluation. Summative evaluation is very expensive, but many aspects of projects can benefit even by small evaluation efforts. Many Division $\mathrm{C}$ members have experience with these efforts that they would be pleased to share.

(ix). The OAD and the projects it supports should be using best practices, and not reinventing all the time. Better communication within the Astronomy Education and Outreach communities would make it easier to identify these best practices.

$(\mathrm{x})$. As the OAD compiles a list of resources it should note that resources should include more than just activities, but also include management advice on things such as how to recruit teachers, how to form teams at a workshop, how to pace a workshop, how to do a needs assessment, how to evaluate the project, and how to do follow-up so that participants feel like part of a community. (Not all of these are appropriate for every project, but for those working with teachers and/or students, or development teams, they are.) 
(xi). The OAD should foster use of Internet for mentoring new practitioners, with recognition that the local person has a better understanding of the local culture.

(xii). In addition to the expert advice from Commission C.C1 (Astronomy Education and Development) and Commission C.C2 (Communicating Astronomy with the Public), the other Commissions in Division C (C.C3 on History of Astronomy and C.C4 on World Heritage and Astronomy) can provide input into helping with the historic and/or cultural links to the projects done within the OAD.

(xiii). Division E provides a forum for observers, theoreticians, modellers and instrumentalists studying a wide range of phenomena related to the structure, radiation and activity of the Sun, the dynamic magnetized solar wind that shapes the heliosphere, and their combined impact on the multitude of bodies within the solar system, including the Earth. The phenomena of solar eclipses also provide a unique chance for professionals, amateurs and the public in general to understand the natural process by providing information on, and facilitating involvement in eclipse research.

(xiv). Division D hosts a Commission on Gravitational Wave Astrophysics, an InterDivision commission on Galaxy Spectral Energy Distribution, one on Supermassive Black Holes, Feedback and Galaxy Evolution and a Supernova Working Group. The strongest assets of Division D which could be of interest of OAD are in general: technology; statistics; x-rays in general (they are well known to the general public even if for different purposes); programming and visualization tools; analytic skills; inspiration. One suggestion would be to create a repository for activities that have been already performed something similar to astroEDU, but more general, with link to useful sites.

(xv). A difficulty is to involve the single individuals of the Division to contribute to $\mathrm{OAD}$ and how to differentiate this from the general outreach activities. How do we collect the inputs from everyone?

(xvi). The description material of OAD is large and well written, however it seems to be lacking of a short and easy description that can be shared with the members to encourage them to participate. This is for the OAD to address.

(xvii). If most of the work has to be done on a voluntary basis, then we need some kind of recognition to give to participants, otherwise it could be difficult to recruit them.

(xviii). A good way of advertising the OAD in the Divisions is through Division Bulletins where applicable.

(xix). Every Division could have a designated liaison with the OAD so that information flows back and forth. A telecon with these Division representatives, say, 2 times per year could keep the links active and information/ideas flowing.

\section{Concluding remarks}

The session proved to be very useful for exploring synergies between the OAD and IAU Divisions. It was clear that greater time was needed to continue the conversation and that the OAD should remain in close communication with points of contact within each Division. The session also seemed to be very useful from the audience perspective with many people expressing appreciation for the brief summaries of what each Division does. There were a number of points that need to be followed up on and from the OAD perspective, we look forward to expanding on these discussions into the future. It was clear that this is an initiative that should be implemented at each General Assembly. 